\title{
Interactive comment on "Relocation of earthquakes in the Southern and Eastern Alps (Austria, Italy) recorded by the dense, temporary SWATH-D network using a Markov chain Monte Carlo inversion” by Azam Jozi Najafabadi et al.
}

\section{Anonymous Referee \#1}

Received and published: 20 January 2021

In this paper, the authors relocate 344 earthquakes in the southern and eastern Alps by exploiting arrival time data from the temporary SWATH-D network, supplemented by Alp-Array stations. Overall, the paper is well written, the methods well explained and tested, and the results carefully obtained and discussed. I suppose one could argue that the work they carried out would normally be integrated into a local earthquake tomography study, in which preliminary location of events is undertaken and a robust $1-D$ reference model is generated prior to the tomography. However, given that this is a new and large dataset from a region of significant interest, that cutting edge methods 
were used for the hypocenter locations and 1-D velocity structure determination, and that the final earthquake distribution does provide insight into active faults in the region, I would be happy to see the paper published following minor revisions.

(1) Line 9: I would be tempted to replace "precise" with "robust".

(2) Line 43: I'm not sure I would say "...they depend not only on proper choices of initial values for hypocenter coordinates..." - what is meant by "proper" in this context - that they are close enough to the "true" location to make the inverse problem locally linear?

(3) Line 44: I don't think I would describe damping as a "technical parameter". It is better described as regularisation.

(4) Lines 115-119: In a way it's a pity that automatic event detection was skipped, since presumably the combined array used in this study provides a much denser data coverage of the region compared to what national seismological agencies have access to. Consequently, there is probably quite a lot of seismicity that has been overlooked, and if the purpose of this paper is to examine earthquake distribution and its relationship to active faulting in the region, then that is somewhat unfortunate. However, on reading the next lines, it appears that an automated picking algorithm was applied, but presumably only to data windowed by the pre-existing catalogue? It would also be interesting to know why the combined national seismological agencies were able to detect and presumably locate 2,639 local events, yet only 384 were deemed good enough for the current study. I understand the issue of seismic gaps and noise, but are the national networks more dense than the network used in this study in some regions?

(5) Line 140: Should be "..and thus is easily mispicked."

(6) Line 175: It is not clear to me how station terms can account for 3-D variations in velocity structure that are not considered in the inversion for 1-D velocity structure.

(7) Line 188: "...for a very large..." - should be "...a very large...."

(8) Lines 188-193: Perhaps I misunderstand something, but the velocity model is de- 
fined by a series of horizontal layers, each with constant $V p$ and $V p / V s$ ? So why does a 3-D Voronoi mesh with $1 \mathrm{~km}$ spacing vertically and horizontally come into it? While

it may be technically correct to use this terminology, isn't it less confusing to describe this as a regular mesh in 3-D with $1 \mathrm{~km}$ spacing? Also, is there any need to correct for Earth's sphericity, since I believe that the Podvin and Lecomte method is in Cartesian coordinates?

Interactive

(9) Lines 193-195: This is perhaps slightly confusing, because the first part seems to indicate an L1 measure of misfit, but with a Gaussian likelihood function, the actual misfit would be L2.

(10) Line 215: It would be interesting to have some numbers on how many iterations constitute the burn-in phase, and how many subsequent iterations were used to build the posterior PDF. (11) Line 263: Should be "earthquake", not "earthquakes".

(11) Line 273: I find it interesting that the Vp uncertainty is almost zero in the $0-20 \mathrm{~km}$ depth range, which the authors put down to dense ray coverage. What values do these uncertainty estimates take, and are they comparable with, say, the standard deviation of the lateral heterogeneity of the synthetic model input at that depth?

(12) Line 284: Should be "Results and discussion".

(13) Line 288: What happens if all model unknowns are allowed to vary in the initial tranche of iterations?

(14) Line 319: Should be "A detailed interpretation of the pattern of corrections...."

(15) Line 323: Should be "Estimation of hypocenter accuracy. ..."

(16) Section 6.3: This section is essentially fine, although with a relatively modest database of 344 earthquakes, it is not entirely clear what new insights are brought to the table beyond what might be gleaned from national catalogues that have been accumulated over periods of decades and involve many more earthquakes (albeit not as well located). To some extent this brings us back to the question of trying to use

Printer-friendly version

Discussion paper 
auto-detection methods that take advantage of this large array to find potentially large numbers of small earthquakes missed by the national agencies.

Interactive comment on Solid Earth Discuss., https://doi.org/10.5194/se-2020-192, 2020. 